\title{
Quantum dynamics of single-photon detection using functionalized quantum transport electronic channels
}

\author{
Catalin D. Spataru* and François Léonard \\ Sandia National Laboratories, Livermore, California 94551, USA
}

(Received 16 November 2018; revised manuscript received 1 August 2019; published 30 August 2019)

\begin{abstract}
Single-photon detectors have historically consisted of macroscopic-sized materials but recent experimental and theoretical progress suggests new approaches based on nanoscale and molecular electronics. Here, we present a theoretical study of photodetection in a system composed of a quantum electronic transport channel functionalized by a photon absorber. Notably, the photon field, absorption process, transduction mechanism, and measurement process are all treated as part of one fully coupled quantum system, with explicit interactions. Using nonequilibrium, time-dependent quantum transport simulations, we reveal the unique temporal signatures of the single-photon detection process, and show that the system can be described using optical Bloch equations, with a new nonlinearity as a consequence of time-dependent detuning caused by the back-action from the transport channel via the dynamical Stark effect. We compute the photodetector signal-to-noise ratio and demonstrate that single-photon detection at high count rate is possible for realistic parameters by exploiting a unique nonequilibrium control of back-action.
\end{abstract}

DOI: 10.1103/PhysRevResearch.1.013018

\section{INTRODUCTION}

Single-photon detection has recently played a key role in addressing long-standing basic physics questions such as Bell's theorem [1] and quantum teleportation [2], as well as enabling potential new approaches for quantum information science [3]. While existing single-photon detectors (e.g., superconducting nanowires, avalanche photodiodes, photomultiplier tubes) show exquisite performance, a fundamental physics question is whether new types of detectors could provide even better performance.

One avenue is to consider arrays of nanometer scale photodetectors instead of the existing bulk detectors. In such arrays, each element simultaneously interacts with the photon field and outputs a signal, providing advantages for performance and photon-number resolution as determined from general considerations [4]. To realize such arrays, it is critical to identify physical device elements that can satisfy the stringent constraints. Several years ago, quantum dot single-photon detectors were first demonstrated and have since achieved good performance [5]. More recently, carbon nanotubes (CNTs) functionalized with molecules have been explored [6-8], as well as photoswitched molecular electronic systems [9]. These nano/molecular systems are promising as array elements but such systems in the presence of lightmatter interactions are out of equilibrium and thus require careful considerations of their properties.

\footnotetext{
*Corresponding author: cdspata@sandia.gov

Published by the American Physical Society under the terms of the Creative Commons Attribution 4.0 International license. Further distribution of this work must maintain attribution to the author(s) and the published article's title, journal citation, and DOI.
}

Previous modeling work focused on the impact of optical absorption by the quantum transport channel itself (e.g., a molecule [10,11], CNT [12], or graphene [13].) Here, we consider a different system where an electronic quantum transport channel (e.g., a CNT) is functionalized with a photon absorber (e.g., a molecule). We study single-photon detection, where the photon field, light-matter interaction, and measurement are coupled as part of one quantum system. We develop a time-dependent, nonequilibrium quantum transport approach for such a system, and reveal the intricate time dynamics of single-photon detection. Furthermore, we demonstrate that the dynamics can be captured using optical Bloch equations (OBEs) with a new nonlinear contribution due to timedependent detuning. Our approach is used to demonstrate a high signal-to-noise ratio for single-photon detection at high count rate $(\sim \mathrm{GHz})$.

\section{DEVICE SYSTEM}

The system is presented schematically in Fig. 1(a). A single-photon coherent state pulse impinges on a quantum transport channel functionalized with a photon absorber. Upon photon absorption, the absorber acquires a permanent dipole moment that creates an electrostatic potential in the transport channel, changing the current. That such a system might be able to detect single photons is not obvious because of quantum back-action. Indeed, because the quantum transport channel interacts coherently with the molecule, measuring the current is akin to performing a continuous quantum measurement on the molecule. Back-action from such quantum measurements have been shown to impact single-photon detection in simplified models [14].

We assume that light absorption takes place in the strongfocusing regime [15-17] with the absorber placed in the focal point of a parabolic mirror and the spatial shape of the pulse 


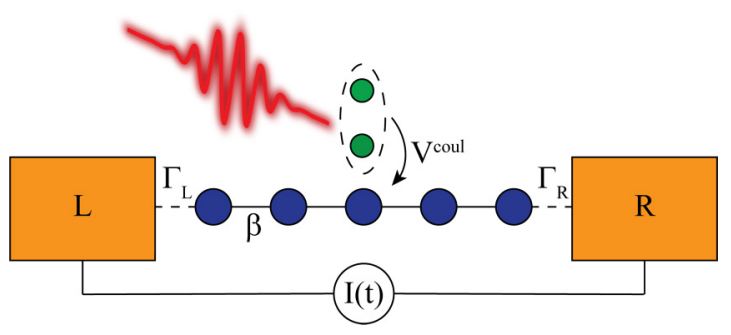

FIG. 1. Sketch of a device consisting of a quantum transport channel functionalized with a photon absorber. Relevant tightbinding parameters are indicated with greek letters. $V^{\text {Coul }}$ is the Coulomb interaction between the absorber and the transport channel.

matching the absorber dipole pattern [18]. The absorber is a diatomic system with one electron and two energy levels: the ground state $(g)$ and the excited state $(e)$. The ground state has no permanent dipole. The photon energy $\hbar \omega$ is resonant with the absorber while the channel is not sensitive to the incoming light pulse (e.g., a small-diameter CNT has well-separated optical absorption peaks due to excitonic effects $[19,20]$; we assume that the molecule optical gap is not matched to these peaks.) This single element detector is narrow band, but an array of such elements could be broadband or even perform energy resolution.

The Hamiltonians $H_{\mathrm{abs}}^{0}$ and $H_{c h}^{0}$ that describe the isolated absorber and transport channel are treated within tight binding (TB). We assume that a single band is relevant for electronic transport. Absorber-channel coupling occurs via the Coulomb interaction, with no charge transfer between them. The transport channel is composed of $N$ sites with intersite distance $a=5$ bohrs and hopping integral $\beta=0.5 \mathrm{eV}$. The left $(L)$ and right $(R)$ leads are modeled in the wide-band limit and their coupling to the channel induces broadenings $\Gamma_{L, R}=\beta$. Parameters for the absorber were chosen to be representative of molecules. To simulate an absorber with a realistic excitedstate radiative lifetime [21], we use $H_{\text {abs }}^{0}=\left[\begin{array}{cc}3 & 2.24 \\ 2.24 & -3\end{array}\right] \mathrm{eV}$ and intersite distance $d_{\mathrm{abs}}=5$ bohrs $\left(d_{\mathrm{abs}}\right.$ determines the strength of the dipole as an electron moves from one absorber site to the other). $H_{\mathrm{abs}}^{0}$ includes the potential from a positive ionic charge distribution that matches the charge distribution of the electron in the bare ground state such that the overall absorber is nonpolar in the ground state. $H_{\mathrm{abs}}^{0}$ results in a bare optical gap $E_{g}^{0}=7.5 \mathrm{eV}$, transition dipole $d_{g e}=3.8$ Debye, and overall permanent dipole in the excited state of 10 Debye. The excited-state spontaneous emission decay rate is $[22,23]$ $\gamma_{\mathrm{rad}} \equiv\left(E_{g}^{0} / \hbar c\right)^{3} d_{g e}^{2} /\left(3 \pi \epsilon_{0}\right)=0.65 \mu \mathrm{eV}$ which yields a radiative lifetime $\tau_{\text {rad }} \equiv \hbar / \gamma_{\text {rad }}=1 \mathrm{~ns}$. This radiative lifetime is the natural reset that determines the count rate of the detector $\sim$ GHz.

The Coulomb potential between absorber and channel is described via the Ohno parametrization $[20,24]$ as $V_{i j}^{\text {Coul }}=$ $U / \sqrt{1+\left[\left(4 \pi \epsilon_{0} / e^{2}\right) U r_{i j}\right]^{2}}$, where $r_{i j}$ is the absorber-channel intersite distance and $U=5 \mathrm{eV}$. The separation between the channel and the farthest absorber site is $d=20$ bohrs, and the permanent dipole is oriented perpendicular to the channel to maximize the impact on electron transport. The Coulomb coupling may lead to near-field nonradiative energy transfer between the absorber and the transport channel, which could prevent the absorber from getting excited. We minimize this effect by having the absorber optical gap significantly larger than the channel band width $4 \beta$, such that no intrachannel excitations are close in energy to the absorber deexcitation energy [25].

We model electrostatic doping of the channel [26] via a fictitious gate potential $V_{G}$ that acts on the onsite energies of the channel. This is equivalent to a potential of opposite sign acting on the leads while leaving the channel unaffected, so the Fermi level inside the two leads depends on $V_{G}$ as well as the applied bias voltage $V_{s d}: E_{F}^{L, R} / e= \pm V_{s d} / 2-V_{G}$. Doping the channel creates a net charge, producing an electric field that slightly polarizes the ground state of the absorber and renormalizes the optical gap of the absorber from $E_{g}^{0}$ to $E_{g}$. The photon energy is $\hbar \omega=E_{g}+\Delta$ with $\Delta$ a possible small detuning.

\section{RESULTS}

\section{A. NEGF dynamics}

To study the system dynamics, we employ a nonequilibrium Green's function (NEGF) formalism based on the equation of motion for the one-particle Green's function [27], treating the Coulomb interaction inside the functionalized transport channel at the mean-field Hartree-Fock level (see Appendix A for formalism details). We also performed extensive calculations where electron correlation effects were included within the $G W$ approximation [28], as discussed in Appendix B.

The equation of motion for the density matrix of the coupled absorber-channel system $\rho$ is projected (i) on the absorber to give $\rho_{\text {abs }}=\left[\begin{array}{ll}\rho_{g g} & \rho_{g e} \\ \rho_{e g} & \rho_{e c}\end{array}\right]$ in the eigenstate basis of $H_{\text {abs }}^{0}$ subject to the initial electric field generated by the doped channel, and (ii) on the channel to give $\rho_{c h}$, a $N \times N$ matrix. Their coupled dynamical equations are

$$
\begin{gathered}
\frac{\hbar \partial \rho_{\mathrm{abs}}}{\partial t}+i\left[H_{\mathrm{abs}}, \rho_{\mathrm{abs}}\right]=\gamma^{\mathrm{rad}}\left[\begin{array}{cc}
\rho_{e e} & -\rho_{g e} / 2 \\
-\rho_{e g} / 2 & -\rho_{e e}
\end{array}\right], \\
\frac{\hbar \partial \rho_{c h}}{\partial t}+i\left\{H_{c h} \rho_{c h}-\text { H.c. }\right\} \\
\quad=\int d \tilde{t}\left\{G_{c h}(t, \tilde{t}) \Sigma_{L+R}^{<}(\tilde{t}-t)+\text { H.c. }\right\} .
\end{gathered}
$$

The (retarded) channel Green's function is given by

$$
G_{c h}(t, \tilde{t})=-i \theta(\tilde{t}-t) \exp \left[-\frac{i}{\hbar} \int_{\tilde{t}}^{t} d t^{\prime} H_{c h}\left(t^{\prime}\right)\right]
$$

while $\Sigma_{L, R}^{<}(t)=i \Gamma_{L, R} \int d E / 2 \pi e^{-i t E} f_{L, R}(E)$ are the lead selfenergies, and $f_{L, R}$ is the Fermi-Dirac electron distribution in the left and right leads. The effective Hamiltonians are

$$
\begin{gathered}
H_{\mathrm{abs}}=H_{\mathrm{abs}}^{0}+\Sigma_{\mathrm{abs}}^{\mathrm{Ha}}(\rho)-e \mathbf{r} \cdot \mathbf{E}(t), \\
H_{c h}=H_{c h}^{0}+\Sigma_{c h}^{\mathrm{Ha}}(\rho)-i \Gamma_{L+R} / 2,
\end{gathered}
$$

where the Hartree self-energy depends self-consistently on $\rho: \Sigma_{i i}^{\mathrm{Ha}}(t)=\sum_{j}^{\mathrm{abs}+c h} \rho_{j j}(t) V_{j i}^{\text {Coul }}$. The last term in Eq. (4) is the coherent field term which depends on the electric field $\mathbf{E}$ associated with the single photon. $\mathbf{E}$ is polarized parallel to the absorber while its temporal shape is determined by the pulse envelope $\xi: E(t)=2 \sqrt{\gamma_{\mathrm{rad}}} / d_{g e} \times e^{i \omega t} \xi(t)$. We choose 


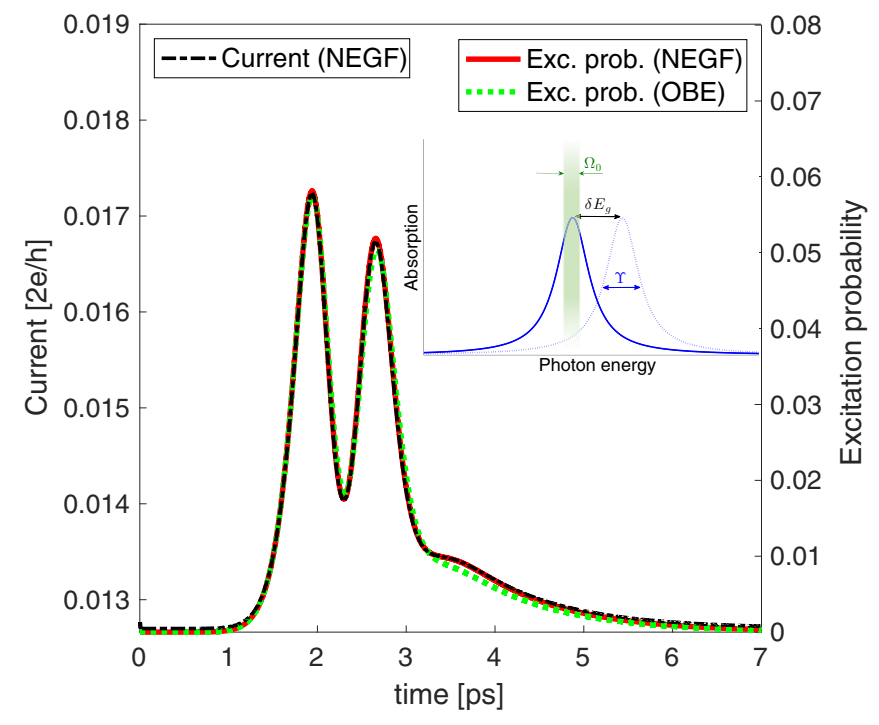

FIG. 2. Calculated time-dependent expectation value of the current through the device (dashed line) and the probability of finding the absorber in the excited state (solid and dotted lines). $N=3$, $\tau^{\mathrm{rad}}=1 \mathrm{ps}, \tau_{0}=417 \mathrm{fs}, t_{c}=2.33 \mathrm{ps}$. The inset illustrates the absorber level detuning via the dynamical Stark effect.

a Gaussian pulse $\xi(t)^{2}=\Omega_{0} / \sqrt{2 \pi} \times \exp \left[-\Omega_{0}^{2}\left(t-t_{c}\right)^{2} / 2\right]$ with bandwidth $\Omega_{0}$ and centered at $t_{c}$. The normalization gives one photon on average in the light pulse.

Because of the computational cost of the NEGF formalism, we first study the case of an absorber with $\tau^{\text {rad }}$ set artificially to $1 \mathrm{ps}$, placed above the middle of a three-site channel $(N=$ 3) subject to $V_{G}=1.125 \mathrm{eV}$ and $V_{s d}=0.5 \mathrm{eV}$. We choose a photon pulse duration $\tau_{0}=\tau_{\text {rad }} / 2.4$. Figure 2 shows the time-dependent expectation value of the current through the device $I(t)$. We use the following expression for the timedependent expectation value of the current from the left and right contacts [29]:

$$
\begin{aligned}
J_{L / R}(t)= & -\frac{e}{\hbar} \operatorname{Tr}\left\{\rho_{c h}(t) \Gamma_{L / R}\right\} \\
& +\frac{e}{\hbar} \int_{\bar{t}} \operatorname{Tr}\left\{\Sigma_{L / R}^{<}(\bar{t}-t) G_{c h}^{r}(t, \bar{t})+\text { H.c. }\right\} .
\end{aligned}
$$

(We note that $J_{L}=-J_{R}$ only in the steady state.) We present results for the symmetrized current $I(t) \equiv\left[J_{L}(t)-J_{R}(t)\right] / 2$, using a smoothing procedure (moving average filter) over a time span equal to a few light cycles. We see in Fig. 2 that the current shows rich temporal behavior, with a clear increase signaling photon detection, but modulated by strong oscillations. Figure 2 also shows the absorber excitation probability $\rho_{e e}(t)$, which correlates with the time-dependent current change $\delta I(t)=I(t)-I_{0}$ via $\delta I(t)=c \times \rho_{e e}(t)$, with a proportionality factor $c$ that can be found via simple steady-state calculations within linear response. The simple relationship between $\rho_{e e}(t)$ and $\delta I(t)$ is due to the fast relaxation times (order of fs) for electrons inside the channel as expected from the magnitude (order of $\mathrm{eV}$ ) of the parameters $\Gamma_{L, R}$ and $\beta$.

The time dependence of the excitation probability can be understood in a simplified picture (see Fig. 2 inset) where the absorption spectrum for the absorber is dynamically modu- lated over timescales $(\sim \mathrm{ps})$ much larger than the light period $(\sim \mathrm{fs})$. In general, a steady-state light field causes power broadening [22,23] giving $\Upsilon=\sqrt{\gamma_{\text {rad }}^{2}+2 \Omega_{\text {Rabi }}^{2}}$ where $\Upsilon$ is the width of the molecular level absorption peak and $\Omega_{\text {Rabi }}=$ $E d_{g e}$ is the Rabi frequency. For a single-photon pulse the upper limit for $\Upsilon$ is $\sqrt{\gamma_{\text {rad }}^{2}+8 / \sqrt{2 \pi} \gamma_{\text {rad }} \Omega_{0}}$. In the isolated absorber case, the optimal pulse bandwidth is [16] $\Omega_{0}=$ $2.4 \times \gamma_{\text {rad }}$ which results in a similar value for $\Upsilon$ and maximizes the overlap between the power broadened absorption line and the bandwidth of the pulse leading to a maximum excitation probability of $\approx 50 \%$. In the coupled case, however, the same choice for pulse duration results in smaller excitation probability, as seen in Fig. 2. This is due to the dynamical Stark effect: as the photon excites the absorber, the associated permanent dipole changes the charge distribution in the transport channel, which in turn changes the electric field acting back on the absorber. The induced time-dependent renormalization $\tilde{E}_{g}(t)=E_{g}+\delta E_{g}(t)$ of the optical gap leads to strong detuning $\hbar \omega-\tilde{E}_{g}(t)=\Delta-\delta E_{g}(t)$ which reduces the absorber excitation. This is illustrated in the inset of Fig. 2 where it is also apparent that a small detuning $\Delta$ matching the mean value of $\delta E_{g}(t)$ may maximize the light-absorber interaction. The time-dependent detuning also explains the oscillating behavior: as the excitation probability and the detuning increase, the dynamics becomes dominated by spontaneous emission which decreases the excitation probability. Thus, the dynamics is governed by a complex interplay of interactions between the absorber and the quantum transport channel.

\section{B. Nonlinear optical Bloch equations}

Having understood the time dependence of $\rho_{e e}(t)$ we now show that we can replace the computationally demanding NEGF approach with a simpler (and computationally faster) description, allowing us to simulate longer timescales. In the case of an isolated absorber, the evolution of the absorber density matrix can be written as optical Bloch equations (OBE) [23,30]

$$
\begin{aligned}
& \frac{\hbar \partial \rho_{e e}}{\partial t}=i \sqrt{\gamma_{\mathrm{rad}}} \xi(t)\left(\tilde{\rho}_{e g}-\tilde{\rho}_{g e}\right)-\gamma_{\mathrm{rad}} \rho_{e e}, \\
& \frac{\hbar \partial \tilde{\rho}_{g e}}{\partial t}=-i \sqrt{\gamma_{\mathrm{rad}}} \xi(t)\left(\rho_{e e}-\rho_{g g}\right)-\left(\frac{\gamma_{\mathrm{rad}}}{2}+i \Delta\right) \tilde{\rho}_{g e}
\end{aligned}
$$

with $\rho_{g g}=1-\rho_{e e}, \tilde{\rho}_{e g}=\tilde{\rho}_{g e}^{*}$, and where we made use of the random wave transformation $\tilde{\rho}_{e g(g e)}=\rho_{e g(g e)} e^{+(-) i \omega t}$. We find that the coupled case can be described by the same set of equations but with $\Delta$ replaced by a time-dependent detuning $\Delta-\delta E_{g}(t)$ that depends on the excitation probability through $\delta E_{g}(t)=f\left(\rho_{e e}(t)\right)$. The function $f$ satisfies $f(0)=0$ and can be easily fitted via a few steady-state calculations. We note that the resulting modified OBE equations are nonlinear due to the term $\delta E_{g} \tilde{\rho}_{g e}$. Figure 2 demonstrates the excellent agreement between the NEGF and OBE approaches. The OBE simulations (employing $\sim 1$ ps time step) in combinations with steady-state calculations of $c$ and $f$ yield a computational time $\sim 5$ orders of magnitude faster than their NEGF counterpart (which uses $\sim 10$ as time step), being thus well suited to search in the multidimensional parameter space for efficient single-photon detection. 


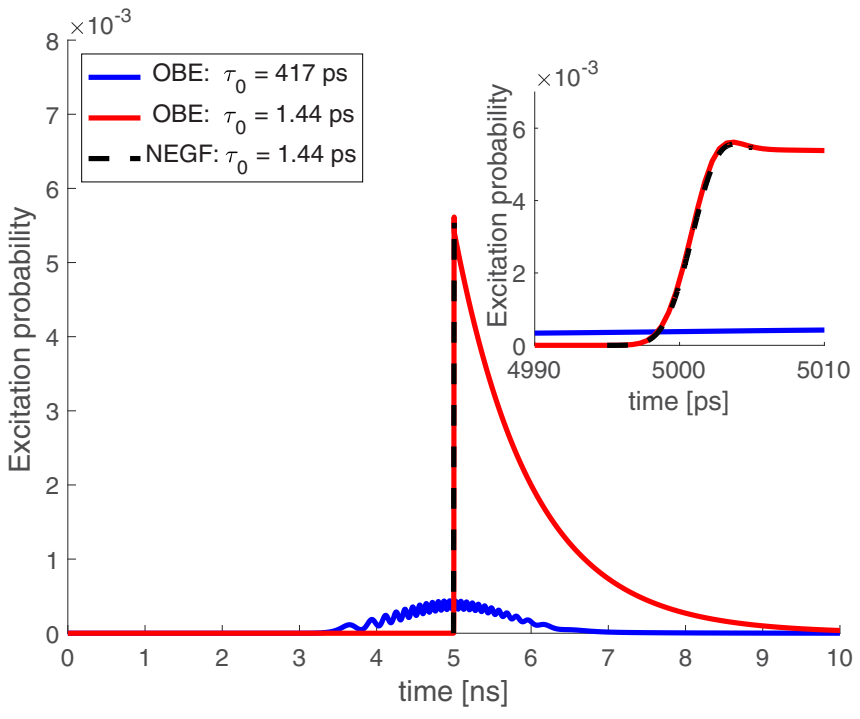

FIG. 3. Absorber excitation probability calculated within OBE for two different pulse durations $\tau_{0}$. NEGF results for the short $\tau_{0}$ are visible in the inset where the propagation time is zoomed in over few ps. $N=3, \tau^{\mathrm{rad}}=1 \mathrm{~ns}, t_{c}=5 \mathrm{~ns}$.

One important parameter is the duration of the light pulse (bandwidth). Indeed, the light-matter coupling is not optimal when the photon bandwidth is much smaller than the optical gap renormalization $\delta E_{g}$. The optimized coupling is realized when the renormalized optical level remains within the bounds set by the photon bandwidth during the course of the light-absorber interaction. This is demonstrated in Fig. 3 which shows OBE results for the excitation probability of the absorber with its actual $\tau_{\text {rad }}=1$ ns coupled to the three-site channel described above, for two different pulse durations: (i) $\tau_{0}=\tau_{\text {rad }} / 2.4=417 \mathrm{ps}$ which is optimal for an isolated absorber; (ii) $\tau_{0}=1.44 \mathrm{ps}$ which is optimal in the coupled case. Case (ii) yields a larger $\Omega_{0}$ and $\Upsilon$ which decreases the impact of detuning (see Fig. 2 inset). One can see that the excitation probability is more than an order of magnitude larger in the latter case. Also shown is the result of a full NEGF calculation, which could only be run for a few ps, but which shows excellent agreement with the OBE calculation. We note that after the pulse passes, the molecule relaxes by spontaneous emission, leading to a short detector reset time $\sim 1$ ns. We find that the parameter $\Delta$ is less impactful since the optimal value increases the excitation probability by $<30 \%$ with respect to the $\Delta=0$ case.

\section{Signal-to-noise ratio}

During the interaction between the photon pulse and the coupled absorber-channel system, the expectation value of the current $I(t)$ changes dynamically from its steady-state value $I_{0}$ prior to light interaction. The main question we ask is as follows: Can the change $\delta I(t)$ be detected? Answering this question requires a calculation of the signal-to-noise ratio (SNR). In this work we consider the situation where the temperature is $T=0$ and where the detector can be operated at high frequency (this is justified below); this implies that the thermal noise and $1 / f$ noise are negligible, so the only source

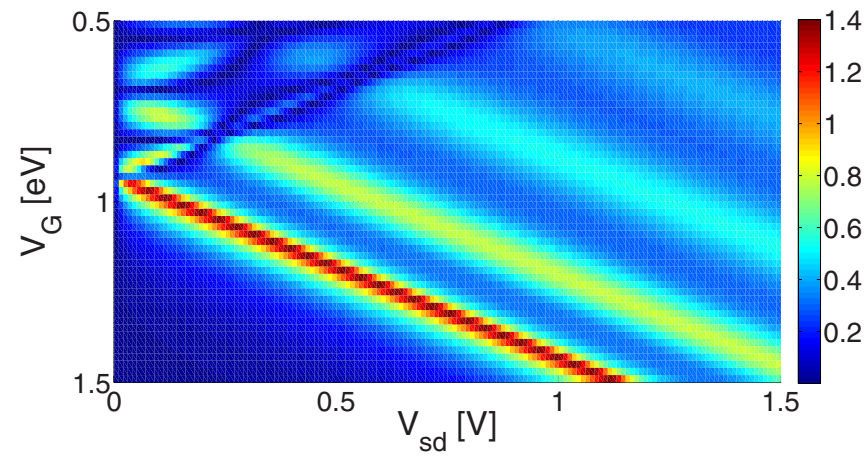

FIG. 4. SNR for an absorber placed above the middle of a sevensite channel. $\tau_{0}$ and $\Delta$ are optimized for each $\left(V_{s d}, V_{G}\right)$ set. The time window is $1.2 \mathrm{~ns}$ and starts when the photon pulse begins interacting with the absorber. $\tau^{\mathrm{rad}}=1 \mathrm{~ns}$.

of noise is the shot noise. Assuming a Poisson distribution of the passing electrons, the root mean square of the current fluctuations in the steady state is $\sigma_{I_{0}}=\sqrt{n_{0}}$ where $n_{0}$ is the integral of $I_{0}$ over a certain time window, i.e., $n_{0}$ is the number of charge carriers that flow in the time window. This also means a conservative value for the Fano factor, namely, $F=1$ [31]. We then define $\mathrm{SNR}=\delta n / \sqrt{n_{0}}$ where $\delta n$ is the integral of $\delta I$ over the time window.

We find that for a given geometry the optimized SNR is achieved for $V_{s d}$ and $V_{G}$ such that one Fermi level (e.g., $E_{F}^{R}$ ) pins the highest prominent peak in the density of states (DOS) of the channel while the other Fermi level is much higher $\left(E_{F}^{L} \gg E_{F}^{R}\right)$. Figure 4 shows $\mathrm{SNR}$ results for the absorber placed above $(d=20$ bohrs $)$ the middle $(j=4)$ of a $N=7$ channel for a range of bias and gate voltages. The peak values in the SNR correlate with resonances in the channel DOS. The overall SNR decreases as $E_{F}^{R}$ moves toward the lowerenergy DOS peaks due to the increasing number of transport eigenchannels inside the bias window that are not impacted by the absorber-channel interaction. For this geometry we obtain an optimized SNR of 1.4 (with $E_{F}^{R}=0.92 \mathrm{eV}, \tau_{0}=372 \mathrm{fs}$, and an optimal time window of $1.2 \mathrm{~ns}$ starting when the photon pulse begins interacting with the absorber).

For efficient photodetection one would like to achieve larger SNR values. Higher SNR is possible through nonequilibrium control of back-action. Indeed, Fig. 5 shows that when the molecule is placed closer to the left electrode $(j=2)$, the change in current is significantly higher and the optimized SNR increases to 3.5 (with $E_{F}^{R}=0.88 \mathrm{eV}$ and $\tau_{0}=10 \mathrm{ps}$ ). The reason for the improved SNR in the asymmetric case is a reduction of the channel back-action on the molecule by a subtle nonequilibrium electronic transport effect. Indeed, the electronic transport is dominated by a narrow energy level (index $N$ ) located near $E_{F}^{R}$. When the molecule is closer to the left electrode, the asymmetric Coulomb perturbation created by the excited molecule increases the coupling between the left lead and the channel level to $\tilde{\Gamma}_{L}^{N}=\Gamma_{L}^{N}(1+\delta)$ and decreases its coupling to the right lead to $\tilde{\Gamma}_{R}^{N} \approx \Gamma_{R}^{N}(1-\delta)$. This results in an increase $\delta \tilde{n}_{c h}$ of the nonequilibrium occupancy [29] of the level which for symmetric leads $\left(\Gamma_{L}^{N}=\Gamma_{R}^{N}\right)$ is $\delta \tilde{n}_{c h} \approx \delta / 2 \int d E\left[f_{L}(E)-f_{R}(E)\right] \operatorname{DOS}(E)$, where the factor $f_{L}-f_{R}$ indicates that $\delta \tilde{n}_{c h} \neq 0$ is essentially a nonequilibrium 


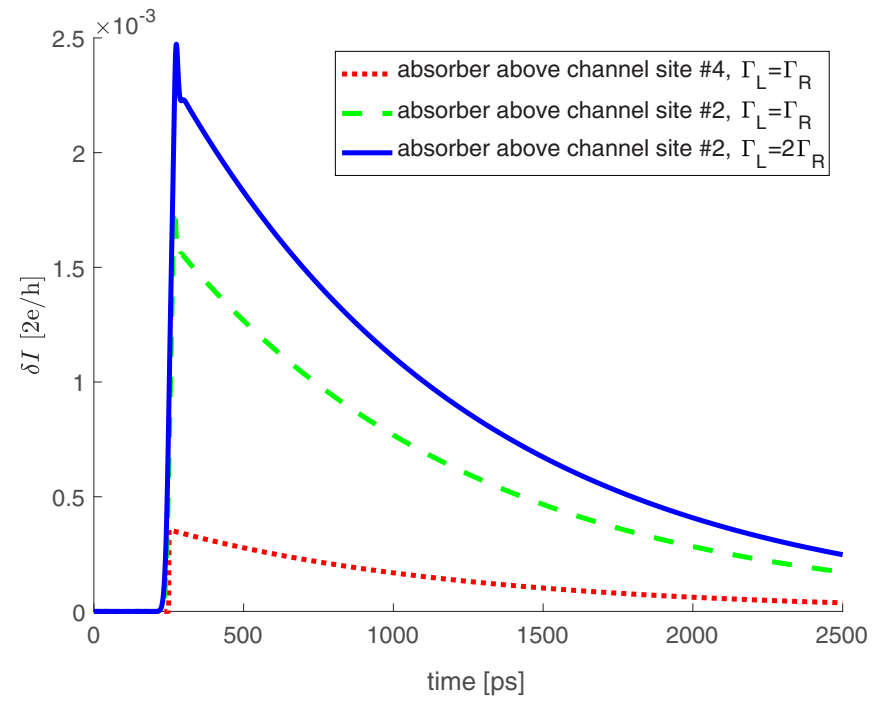

FIG. 5. OBE results for the change in current $\delta I(t)$ for three different design strategies. $N=7, E_{F}^{L} \gg E_{F}^{R}, \tau^{\mathrm{rad}}=1 \mathrm{~ns}, t_{c}=250 \mathrm{ps}$. The value of the current prior to light interaction is $I_{0}[2 e / h]=0.013$, 0.043 , and 0.054 , respectively.

effect. This effect opposes the more general decrease in level occupancy $\delta \bar{n}_{c h}$ generated when the electron in the absorber gets closer to the channel pushing away its electrons into the leads (as illustrated in Fig. 6). Thus, the electric field causing detuning of the absorber is smaller in the asymmetric case, ultimately allowing much higher excitation probability $(3.3 \%$ for $j=2$ versus $0.17 \%$ for $j=4$ ).
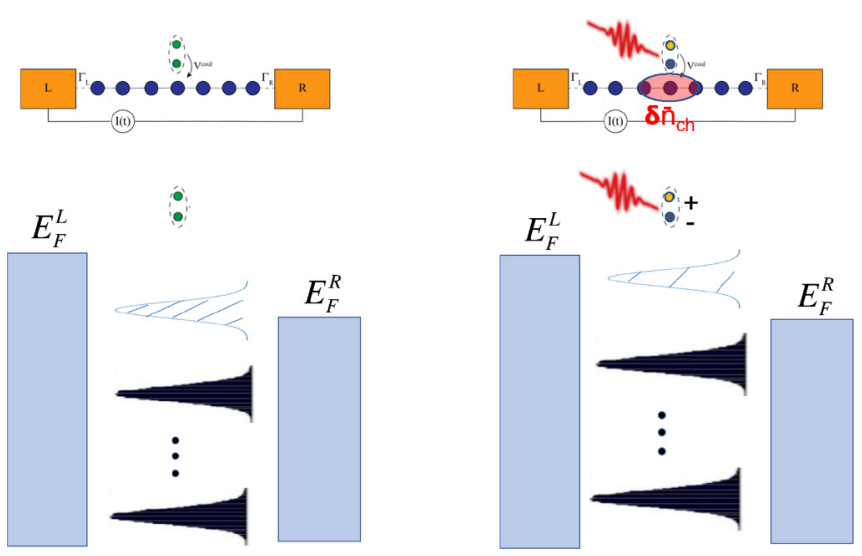

FIG. 6. Upper panels: sketch of the functionalized channel device before (left) and during (right) photoexcitation. Lower panels: energy-level alignment between channel and leads for a possible bias and gate voltage configuration where only the highest-energy channel level contributes to the channel current and is positioned near the bias window. Partial level occupancy is indicated with hashing. As the channel energy levels shift up upon absorber photoexcitation (right panel), the electron occupancy of the highest-energy level decreases, as indicates with less hashing. The resulting depopulation $\delta \bar{n}_{c h}$ in the channel electronic density modifies the electric field induced by the channel and impacting the absorber. (Figure not drawn to scale: the actual shift of energy levels is much smaller than the energy spacing between levels.)
Further increase of SNR is possible in configurations where the leads are asymmetrically coupled to the channel before photoexcitation. Indeed, as seen in Fig. 5, a larger change in current is achieved by coupling the level more strongly to the left lead by making $\Gamma_{L}=2 \Gamma_{R}=1 \mathrm{eV}$. For the optimal parameters $E_{F}^{R}=0.86 \mathrm{eV}$ and $\tau_{0}=12 \mathrm{ps}$ the absorber excitation probability is $4.0 \%$ and during the $1.2-n s$ time window there are about 800 more electrons passing through the device, leading to an SNR of 4.5.

\section{CONCLUSION}

In conclusion, we performed quantum-mechanical calculations of single-photon detection for an absorber coupled to a quantum transport channel via the Coulomb interaction. Notably, our work considers the coherent photon field, optical absorption process, and transduction mechanism as part of one coupled quantum system. We reveal the complex dynamics of such systems, and identify nonequilibrium electronic transport as a mechanism to control back-action. With a purely electronic coupling between the absorber and transport channel, we find a signal-to-noise ratio larger than 4 and with reset time of $\sim 1$ ns which suggests that single-photon detection is possible with a minimal device design. This SNR arises for optimal pulse durations of $\sim 10 \mathrm{ps}$; it is possible to increase the optimal pulse duration by decreasing the absorber-channel coupling (e.g., increasing the separation), or by using absorbers with longer spontaneous emission decays at the cost of lower count rate. We anticipate rich additional physics in these systems, for example, through considerations of current fluctuations and full counting statistics of electrons [32-34]. More broadly, we envision these systems as part of large arrays of nanoscale detection elements, all simultaneously interacting with the photon field.

\section{ACKNOWLEDGMENTS}

We thank M. Sarovar for useful discussions. Work supported by the Defense Advanced Research Projects Agency (DARPA) DETECT program. The views, opinions, and/or findings expressed are those of the author and should not be interpreted as representing the official views or policies of the Department of Defense or the US Government. Sandia National Laboratories is a multimission laboratory managed and operated by National Technology and Engineering Solutions of Sandia, LLC., a wholly owned subsidiary of Honeywell International, Inc., for the US Department of Energy's National Nuclear Security Administration under Contract No. DE-NA-0003525.

\section{APPENDIX A: QUANTUM KINETIC EQUATION FOR THE ONE-PARTICLE DENSITY MATRIX}

Here, we derive the quantum kinetic equation for the one-particle charge density matrix $\rho$ of the functionalized transport channel interacting with a single-photon coherent light field. We use a nonequilibrium Green's function (NEGF) approach for the one-particle Green's function:

$$
G(1,2)=-i\left\langle T_{C}\left[\Psi_{H}(1) \Psi_{H}^{\dagger}(2)\right]\right\rangle,
$$


where $G$ is the Green's function, $\Psi$ is the system wave function for Hamiltonian $H$, and $T_{C}$ denotes time ordering along the double-time Keldysh contour [35]. The arguments for $G$ denote temporal, spatial, and spin degrees of freedom. For simplicity, next we show explicitly only the time arguments. One starts from the Dyson equation

$$
\left(G_{0}^{-1}-\Sigma\right) G=I
$$

where the self-energy $\Sigma$ accounts for electron-electron (Coulomb) interactions inside the functionalized transport channel as well as for the coupling of the transport channel to the leads. In this equation, $G_{0}$ is the Green's function for the isolated functionalized channel in the absence of leads. $G_{0}^{-1}$ is diagonal in the time domain (for simplicity we set $\hbar=1$ )

$$
G_{0}^{-1}\left(t_{1}, t_{2}\right)=\delta\left(t_{1}-t_{2}\right)\left[i \partial t_{1}-H_{\text {tot }}^{0}-U\left(t_{1}\right)\right]
$$

with $H_{\mathrm{tot}}^{0}=H_{\mathrm{abs}}^{0}+H_{c h}^{0}$ the single-particle time-independent Hamiltonian of the functionalized channel (bare, with no electron-electron interation). $U(t)$ is the radiation field which at this point is treated classically within the dipole approximation [36]: $U(t)=-\vec{d} \cdot \vec{E}(t)$ where $\vec{d} \equiv e \vec{r}$ is the dipole operator.

The Dyson equation leads to the Kadanoff-Baym equations (KBEs), a set of coupled integrodifferential equations for the lesser/greater/advanced/retarded Green's functions involving two-time arguments $G^{</>/ a / r}\left(t_{1}, t_{2}\right)$. Using the Langreth rules [37] for analytic continuation, the KBEs read as [38,39]

$$
\begin{gathered}
{\left[i \partial t_{1}-H_{\mathrm{tot}}^{0}-U\left(t_{1}\right)\right] G^{r}\left(t_{1}, t_{2}\right)} \\
=\delta\left(t_{1}-t_{2}\right)+\int d t \Sigma^{r}\left(t_{1}, t\right) G^{r}\left(t, t_{2}\right), \\
{\left[i \partial t_{1}-H_{\mathrm{tot}}^{0}-U\left(t_{1}\right)\right] G^{<}\left(t_{1}, t_{2}\right)} \\
=\int d \bar{t}\left\{\Sigma^{r}\left(t_{1}, \bar{t}\right) G^{<}\left(\bar{t}, t_{2}\right)+\Sigma^{<}\left(t_{1}, \bar{t}\right) G^{a}\left(\bar{t}, t_{2}\right)\right\}, \\
{\left[-i \partial t_{2}-H_{\mathrm{tot}}^{0}-U\left(t_{2}\right)\right] G^{<}\left(t_{1}, t_{2}\right)} \\
=\int d \bar{t}\left\{G^{r}\left(t_{1}, \bar{t}\right) \Sigma^{<}\left(\bar{t}, t_{2}\right)+G^{<}\left(t_{1}, \bar{t}\right) \Sigma^{a}\left(\bar{t}, t_{2}\right)\right\},
\end{gathered}
$$

and similar equations for the other Green's function components [38,39].

Subtracting Eqs. (A5) and (A6) and setting $t_{1}=t_{2}=t$ one obtains the generalized Kadanoff-Baym (GKB) equation $[40,41]$ for $\rho(t) \equiv\left\langle\Psi_{H}^{\dagger}(t) \Psi_{H}(t)\right\rangle=-i G^{<}(t, t)$ :

$$
\begin{aligned}
\frac{\partial \rho}{\partial t}+ & i\left[H_{\mathrm{tot}}^{0}+U(t), \rho(t)\right]_{-} \\
= & \int_{t_{0}}^{t} d \bar{t}\left\{\Sigma^{<}(t, \bar{t}) G^{a}(\bar{t}, t)-G^{r}(t, \bar{t}) \Sigma^{<}(\bar{t}, t)\right. \\
& \left.+\Sigma^{r}(t, \bar{t}) G^{<}(\bar{t}, t)-G^{<}(t, \bar{t}) \Sigma^{a}(\bar{t}, t)\right\}
\end{aligned}
$$

with the initial condition formulated at the reference time $t_{0}$.

So far, the GKB equation does not represent a closed equation for $\rho$ because the right-hand side of Eq. (A7) involves Green's functions with two different time arguments. Solving the $\mathrm{KBE}$ for $G^{</ r}$ via a two-time propagation approach is a formidable task which has been achieved only in very simple cases within certain approximations for $\Sigma$. Most studies rely on an approximation [e.g., GKB ansatz (GKBA) [42]] to reconstruct two-time Green's functions from $\rho$ which results in closure of the GKB equation. In this work we do not need to invoke the GKBA as we achieve single-time propagation of $\rho$ using the following simplifications. First, we treat Coulomb interaction effects via first-order perturbation in the Coulomb potential, i.e., at the mean-field Hartree-Fock level [43]. Second, we consider that the leads are well described by the wide-band limit. These two approximations simplify the self-energy expressions

$$
\begin{gathered}
\Sigma^{r / a}(t, \bar{t})=\left[\Sigma^{\mathrm{Ha}}(t) \mp i\left(\Gamma_{L}+\Gamma_{R}\right) / 2\right] \delta(t, \bar{t}), \\
\Sigma^{<}(\bar{t}, t)=\Sigma_{L}^{<}(\bar{t}-t)+\Sigma_{R}^{<}(\bar{t}-t),
\end{gathered}
$$

where $\Sigma_{L / R}^{<}$are simply related to the Fourier transform of the Fermi-Dirac distribution functions in the left $(L)$ and right $(R)$ leads via the energy-independent broadenings $\Gamma^{L / R}$ induced by the contacts [29]

$$
\Sigma_{L / R}^{<}(\tau)=i \Gamma^{L / R} \int \frac{d E}{2 \pi} e^{-i E \tau} f_{L / R}(E) .
$$

The Hartree self-energy depends self-consistently on the sitediagonal part of $\rho$ and reads as (in the site basis)

$$
\Sigma_{i i}^{\mathrm{Ha}}(t)=\sum_{j} \rho_{j j}(t) V_{j i}^{\mathrm{Coul}} .
$$

The aforementioned simplifications result in closure of the GKB equation [27]

$$
\begin{gathered}
\frac{\partial \rho}{\partial t}+i\left\{\left[H_{\mathrm{tot}}^{0}+\Sigma^{\mathrm{Ha}}(t)+U(t)-i\left(\Gamma_{L}+\Gamma_{R}\right) / 2\right] \rho(t)-\text { H.c. }\right\} \\
=-\int_{t_{0}}^{t} d \bar{t}\left\{G^{r}(t, \bar{t})\left[\Sigma_{L}^{<}(\bar{t}-t)+\Sigma_{R}^{<}(\bar{t}-t)\right]+\text { H.c. }\right\}
\end{gathered}
$$

where $G^{r}$ obeys the following simplified version of the nonequilibrium Dyson equation (A4):

$$
\begin{aligned}
& {\left[i \partial t_{1}-H_{\mathrm{tot}}^{0}-\Sigma^{\mathrm{Ha}}\left(t_{1}\right)-U\left(t_{1}\right)-i\left(\Gamma_{L}+\Gamma_{R}\right) / 2\right] G^{r}\left(t_{1}, t_{2}\right)} \\
& \quad=\delta\left(t_{1}-t_{2}\right)
\end{aligned}
$$

with solution

$$
\begin{aligned}
G^{r}(t, \bar{t})= & -i \theta(t-\bar{t}) \exp \left\{-i \int_{\bar{t}}^{t} d t^{\prime}\left[H_{\mathrm{tot}}^{0}+U\left(t^{\prime}\right)\right.\right. \\
& \left.\left.+\Sigma^{\mathrm{Ha}}\left(t^{\prime}\right)-i\left(\Gamma_{L}+\Gamma_{R}\right) / 2\right]\right\} .
\end{aligned}
$$

It is convenient to take advantage of the absence of charge transfer between absorber and transport channel. For that, we first note that if no initial correlations exist between the two subspaces at the initial time $t_{0}$, then one has at any subsequent time $t$

$$
\rho(t)=\left[\begin{array}{cc}
\rho_{\mathrm{abs}}(t) & 0 \\
0 & \rho_{c h}(t)
\end{array}\right] .
$$


We also note that the broadenings $\Gamma^{L / R}$ exist only in the channel subspace. Projecting Eqs. (A12) and (A14) on the absorber and transport channel subspaces we obtain

$$
\begin{gathered}
\frac{\partial \rho_{\mathrm{abs}}}{\partial t}+i\left[H_{\mathrm{abs}}^{0}+\Sigma_{\mathrm{abs}}^{\mathrm{Ha}}(t)+U(t), \rho_{\mathrm{abs}}(t)\right]_{-}=0, \\
\frac{\partial \rho_{c h}}{\partial t}+i\left\{\left[H_{c h}^{0}+\Sigma_{c h}^{\mathrm{Ha}}(t)-i\left(\Gamma_{L}+\Gamma_{R}\right) / 2\right] \rho_{c h}(t)-\text { H.c. }\right\} \\
=-\int_{t_{0}}^{t} d \bar{t}\left\{G_{c h}^{r}(t, \bar{t})\left[\Sigma_{L}^{<}(\bar{t}-t)+\Sigma_{R}^{<}(\bar{t}-t)\right]+\text { H.c. }\right\}
\end{gathered}
$$

$$
\begin{aligned}
G_{c h}^{r}(t, \bar{t})= & -i \theta(t-\bar{t}) \exp \left\{-i \int_{\bar{t}}^{t} d t^{\prime}\left[H_{c h}^{0}+\Sigma_{c h}^{\mathrm{Ha}}\left(t^{\prime}\right)\right.\right. \\
& \left.\left.-i\left(\Gamma_{L}+\Gamma_{R}\right) / 2\right]\right\}
\end{aligned}
$$

Equation (A16) shows that the electron dynamics in the absorber differs from the isolated absorber case only through the time-dependent potential $\Sigma_{\text {abs }}^{\mathrm{Ha}}(t)$ generated by the charge in the channel in response to self-consistent changes in the absorber charge density upon photoexcitation. The absorber dynamics is thus effectively equivalent to that of an isolated two-level system subject to a modulated (no longer monochromatic) light field.

So far, we have treated the radiation field classically. In the case of coherent light (as opposed to a Fock state) this classical treatment, together with a proper normalization of the electric field entering the expression for $U(t)$, accounts for the absorbtion and stimulated emission components [16,17] of the light-absorber interaction. However, the spontaneous emission component, which cannot be treated classically, is crucial and needs to be accounted for in the context of single-photon detection. In order to introduce it, we note that this component should describe the decay of the absorber excited state to the ground state via spontaneous emission of radiation into the environment (vacuum) independently of the occupation of the quantized states of the electromagnetic environment. It is thus sufficient to consider the case of an isolated absorber in the absence of a light pulse. In this case, the complete dynamics can be obtained via the masterequation approach [17] or from optical Bloch equations [23] and it yields the following kinetic equation:

$$
\frac{\partial \rho_{\mathrm{abs}}}{\partial t}+i\left[H_{\mathrm{abs}}^{0}, \rho_{\mathrm{abs}}(t)\right]_{-}=\gamma^{\mathrm{rad}}\left[\begin{array}{cc}
\rho_{e e} & -\rho_{g e} / 2 \\
-\rho_{e g} / 2 & -\rho_{e e}
\end{array}\right],
$$

where $\gamma^{\text {rad }}$ denotes the radiative decay rate of the absorber excited state and we used the following notation for $\rho_{\text {abs }}$ in the absorber ground- $(g)$ and excited- $(e)$ state basis: $\rho_{\mathrm{abs}} \equiv$ $\left[\begin{array}{cc}\rho_{g g} & \rho_{g e} \\ \rho_{e g} & \rho_{e e}\end{array}\right]$.

By comparing Eqs. (A16) and (A19), it follows that the right-hand side of Eq. (A19) represents the spontaneous emission term. We can now include this term in the more general case that describes the absorber interacting with the transport channel and the coherent light pulse [Eq. (A16)], with the final expression reading as

$$
\begin{aligned}
& \frac{\partial \rho_{\mathrm{abs}}}{\partial t}+i\left[H_{\mathrm{abs}}^{0}+\Sigma_{\mathrm{abs}}^{\mathrm{Ha}}(t)+U(t), \rho_{\mathrm{abs}}(t)\right]_{-} \\
& \quad=\gamma^{\mathrm{rad}}\left[\begin{array}{cc}
\rho_{e e} & -\rho_{g e} / 2 \\
-\rho_{e g} / 2 & -\rho_{e e}
\end{array}\right] .
\end{aligned}
$$

The coupled equations (A17) and (A20) represent the quantum kinetic equation for the functionalized transport channel which we solve via the Runge-Kutta (RK4) method [44] for time propagation. We note that our derivation does not rely on perturbation theory expansion about the absorber-light field coupling.

\section{APPENDIX B: ELECTRON CORRELATION EFFECTS WITHIN THE $G W$ APPROXIMATION}

In this Appendix, we discuss the impact of electron correlation and image charge effects on our results. Our approach for solving the quantum kinetic equation for the functionalized transport channel is based on the time-dependent HartreeFock (TDHF) level of theory. Since it is known that electron correlation effects beyond TDHF can be important [27,45], we address below the question of whether these effects may alter the conclusion of our work, namely, that single-photon detection can be achieved with a functionalized transport channel device under optimal operating conditions. Electron correlation effects play different roles in different parts of the functionalized transport channel:

(1) Electron transport channel subspace. The electronic properties of the transport channel are expected to be impacted by inclusion of electron correlation effects inside the channel subspace. These effects may alter the quasiparticle energy levels of the channel as well as their energy broadening, which may lead to significant changes in the channel current [27] especially when screening effects in the channel are important.

(2) Interaction between channel and absorber. Electron correlation effects between the transport channel and the absorber are also important as they are expected to affect the absorber optical level via image-charge effects.

(3) Absorber subspace. Electron correlation effects inside the absorber subspace are not important because no charge transfer is allowed between absorber and channel and the absorber only accommodates a single electron. As discussed in Appendix A, the absorber dynamics at the Hartree-Fock level is effectively equivalent to that of an isolated singleelectron system subject to a modulated light field. Because in this case there are no Coulomb interactions inside the absorber subspace, treating the Coulomb interaction within first-order perturbation theory (i.e., within Hartree-Fock) has no impact on the quantum dynamics of the coupled system, as numerically verified. Higher orders in perturbation theory inside this subspace are thus not expected to play an important role.

\section{Electron correlation effects in the transport channel}

We calculated the impact of electron correlations in the channel by including them at the $G W$ level [28] during the steady-state calculations that determine the constant $c$ and function $f$ characterizing the absorber action/channel 

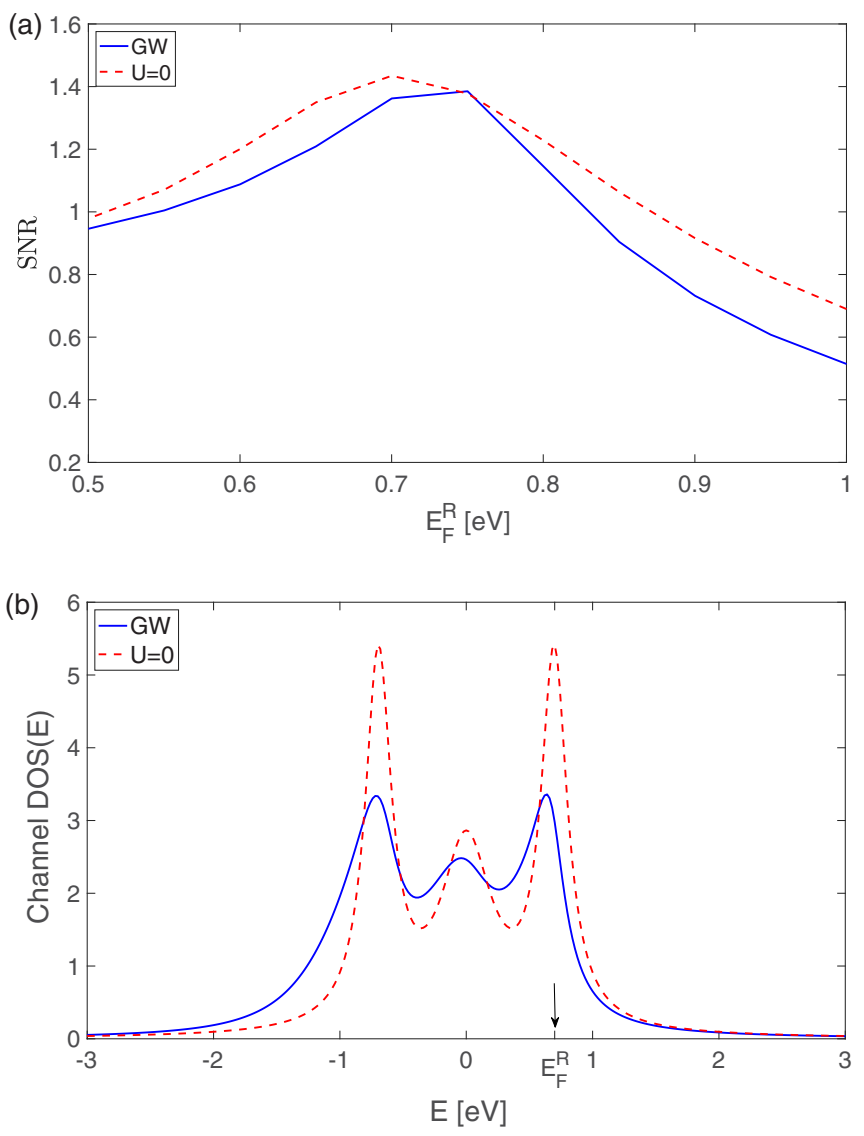

FIG. 7. (a) Optimized SNR for an absorber placed above the middle of a three-site channel. $E_{F}^{L}$ is well above the channel energy levels and the pulse duration $\tau_{0}$ is optimized for each $E_{F}^{R}$ value. The time window is $1.2 \mathrm{~ns}$ and starts when the photon pulse begins interacting with the absorber. $\tau^{\text {rad }}=1$ ns. $N=3, j=2, d=$ 20 bohrs. The Coulomb interaction inside the channel is either neglected $(U=0)$ or treated at the $G W$ level. (b) Electronic DOS of the channel for the case of $E_{F}^{R}=0.7 \mathrm{~V}$ (indicated by arrow).

back-action effects discussed in the main text. We compare the SNR results for two cases:

(i) Neglecting Coulomb interactions in the channel ( $U$ set to zero inside the channel). We have used this approximation throughout the results presented in the main text as it allows a gain in the computational time of several orders of magnitude over the case where Coulomb interactions in the channel are included.

(ii) Electron self-energy effects in the channel considered at the $G W$ level. We have implemented these effects by extending to the multisite channel case a $G W$ approach [46-48] previously applied by one of us to the single-site channel case. In this case we considered a spin-dependent Coulomb interaction screened by an average screening constant $[49,50]$ $\kappa=\left(\epsilon_{\text {vacuum }}+\epsilon_{\text {substrate }}\right) / 2$. Assuming a conducting channel on an $\mathrm{Al}_{2} \mathrm{O}_{3}$ gate oxide we set $\epsilon_{\text {substrate }}=9$ which yields $\kappa=5$.

The SNR results for the two cases are shown in Fig. 7(a) for an absorber above the central site of a three-site conducting channel. The lead Fermi levels are chosen such that one of them $\left(E_{F}^{L}\right)$ is well above the energy levels in the channel while the other one $\left(E_{F}^{R}\right)$ is near the highest energy level: the optimal SNR is obtained precisely in this typical configuration where the doping level in the channel is small [one Fermi level pinning the highest peak in the electronic DOS of the channel, see Fig. 7(b)]. The comparison shows that screening effects inside the conducting channel (that are captured at the $G W$ level) play a minor effect on the optimized SNR (with maximum value of about 1.4), leading to an insignificant difference between the two cases. This is due to the relatively low doping levels (hence screening effects are small) as well as the fact that the induced changes (due to screening inside the channel) in the absorber action and channel back-action tend to cancel each other. Based on these results, we expect that the main conclusion of our work (that it is possible to achieve large SNRs under optimal conditions) is not affected by the neglect of Coulomb interactions inside the channel.

\section{Electron correlation effects due to absorber-channel interactions \\ a. Image-charge effects in the linear response regime}

In the linear-reponse regime (i.e., small absorber excitation probability [51]) and for an absorber with permanent dipole in the excited state, the absorber optical level is renormalized via the image-charge effect originating from the interaction between the absorber excited-state permanent dipole and its image in the channel [52-54]. This effect is not captured within time-dependent Hartree-Fock and to account for it via steady-state calculations one needs to include electron correlation effects (e.g., within the $G W$ approximation) in the coupled absorber-channel space [55]. To good approximation it reads as [52]

$$
\Delta E_{g}^{i m} \approx 1 / 2\left\langle\phi_{e}^{*} \phi_{e}-\phi_{g}^{*} \phi_{g}\left|w_{c h}-v\right| \phi_{e}^{*} \phi_{e}-\phi_{g}^{*} \phi_{g}\right\rangle,
$$

where $w_{c h}$ is the static screened Coulomb interaction of the channel alone and $w_{c h}-v$ represents the image potential due to the channel.

The image-charge effect described by $\Delta E_{g}^{i m}$ affects the light-absorber resonant condition in the linear response regime. However, capturing quantitatively the renormalized absorber optical gap in the linear response regime is not essential since we assume in the main text that resonant lightabsorber coupling is achieved by adjusting the photon energy to match the absorber optical gap. Adding the contributions $\Delta E_{g}^{i m}$ would only mean that the optimal SNR would occur at a slightly different photon energy, but would not affect the value of the SNR or the dynamics of the system.

\section{b. Image-charge effects in the nonlinear response regime}

The regime addressed by our simulations belongs to the nonlinear response where additional time-dependent renormalization of the absorber optical gap occurs. It is critical to capture this dynamical effect as it determines the back-action from the channel to the absorber and affects the quantum dynamics of the system. There are two main contributions to the dynamical renormalization of the absorber optical gap:

(I) the dynamical Stark effect, denoted in the main text as $\delta E_{g}(t)$ 
(II) time-dependent contributions to $\Delta E_{g}^{i m}$, which we denote here as $\delta E_{g}^{i m}(t)$.

We argue below that contribution (I) (captured by our simulations described in the main text) is the dominant one in the nonlinear response regime.

For a qualitative comparison it is useful to express $\delta E_{g}(t)$ as an image-charge effect, distinct from the one described in Appendix B 2 a. To see this, consider the case where the absorber excitation probability has increased at time $t$ to $\mathcal{P}(t)$. In practice, $\mathcal{P}(t)$ is small (because of channel back-action it only reaches values of a few \%), hence, in the analysis below it is sufficient to consider linear expansion about $\mathcal{P}(t)$. Since the absorber acquires a partial permanent dipole $\mathcal{P}(t) \times \mu_{\text {perm }}$ and an associated change in its charge distribution $\delta \rho_{\text {abs }}(t)=$ $\mathcal{P}(t)\left[\phi_{e}^{*} \phi_{e}-\phi_{g}^{*} \phi_{g}\right]$, it creates an "image"-charge perturbation $\delta \rho_{c h}(t)$ in the conducting channel. This leads to a new type of absorber optical gap renormalization (with respect to $t=0$, i.e., with respect to the linear response regime [56]) that originates from a change in the Hartree self-energy $\delta \Sigma_{\text {abs }}^{\mathrm{Ha}} \equiv$ $\Sigma_{\mathrm{abs}}^{\mathrm{Ha}}\left(\rho+\delta \rho_{c h}\right)-\Sigma_{\mathrm{abs}}^{\mathrm{Ha}}(\rho)$. To see this, let us consider the renormalization of the HOMO/LUMO $(g / e)$ absorber energy levels $\epsilon_{g / e}$ :

$$
\delta \epsilon_{g / e}(t)=\left\langle\phi_{g / e}\left|\delta \Sigma_{\mathrm{abs}}^{\mathrm{Ha}}(t)\right| \phi_{g / e}\right\rangle=\left\langle\delta \rho_{c h}(t)|v| \phi_{g, e}^{*} \phi_{g, e}\right\rangle .
$$

We use the fact that the interaction between a test charge distribution $\left|\phi_{g, e}\right|^{2}$ and the charge distribution $\delta \rho_{\text {abs }}(t)$ in proximity to the transport channel is mediated by $w_{c h}$, leading to the electrostatic potential felt by the test charge distribution: $\left\langle\delta \rho_{\text {abs }}(t)\left|w_{c h}\right|\left|\phi_{g, e}\right|^{2}\right\rangle$. Using the method of images, this potential can be rewritten as the sum between the electrostatic potential created by $\delta \rho_{\text {abs }}(t)$ alone $\left\langle\delta \rho_{\text {abs }}(t)|v|\left|\phi_{g, e}\right|^{2}\right\rangle$ and the electrostatic potential created by the image-charge perturbation $\delta \rho_{c h}(t):\left\langle\delta \rho_{c h}(t)|v|\left|\phi_{g, e}\right|^{2}\right\rangle$. Equating these allows us to write

$$
\begin{aligned}
\delta \epsilon_{g / e}(t) & \left.=\left\langle\delta \rho_{\mathrm{abs}}(t)\right)\left|w_{c h}-v\right|\left|\phi_{g, e}\right|^{2}\right\rangle \\
& =\mathcal{P}(t)\left\langle\left|\phi_{e}\right|^{2}-\left|\phi_{g}\right|^{2}\left|w_{c h}-v\right|\left|\phi_{g, e}\right|^{2}\right\rangle
\end{aligned}
$$

and the corresponding renormalization of the absorber optical gap

$$
\delta E_{g}(t) \equiv \delta \epsilon_{e}(t)-\delta \epsilon_{g}(t)=\left\langle\delta \rho_{c h}(t)|v|\left|\phi_{e}\right|^{2}-\left|\phi_{g}\right|^{2}\right\rangle
$$

is written as

$$
\delta E_{g}(t)=\mathcal{P}(t)\left\langle\left|\phi_{e}\right|^{2}-\left|\phi_{g}\right|^{2}\left|w_{c h}-v\right|\left|\phi_{e}\right|^{2}-\left|\phi_{g}\right|^{2}\right\rangle .
$$

Let us focus next on the image-charge effect not included in our simulations $\delta E_{g}^{i m}(t)$, and see how it compares with $\delta E_{g}(t)$. This change originates from the dynamical renormalization [due to $\delta \rho_{\text {abs }}(t)$ ] of the channel screened Coulomb interaction $\delta w_{c h}(t) \equiv w_{c h}(t)-w_{c h}$. Taking this into account, Eq. (B1) yields

$$
\delta E_{g}^{i m}(t) \approx 1 / 2\left\langle\left|\phi_{e}\right|^{2}-\left|\phi_{g}\right|^{2}\left|w_{c h}(t)-w_{c h}\right|\left|\phi_{e}\right|^{2}-\left|\phi_{g}\right|^{2}\right\rangle .
$$

We also expand $w_{c h}(t)$ to first order in $\mathcal{P}(t): w_{c h}(t)=w_{c h}+$ $\mathcal{P}(t) \dot{w}$ where $\dot{w} \equiv \frac{\delta w_{c h}(t)}{\delta \mathcal{P}(t)}$ is a generic quantity that depends on the absorber-channel separation $d$ and satisfies $\dot{w}(d \rightarrow \infty)=$ 0 . This leads to

$$
\delta E_{g}^{i m}(t) \approx 1 / 2 \mathcal{P}(t)\left\langle\left|\phi_{e}\right|^{2}-\left|\phi_{g}\right|^{2}|\dot{w}|\left|\phi_{e}\right|^{2}-\left|\phi_{g}\right|^{2}\right\rangle .
$$
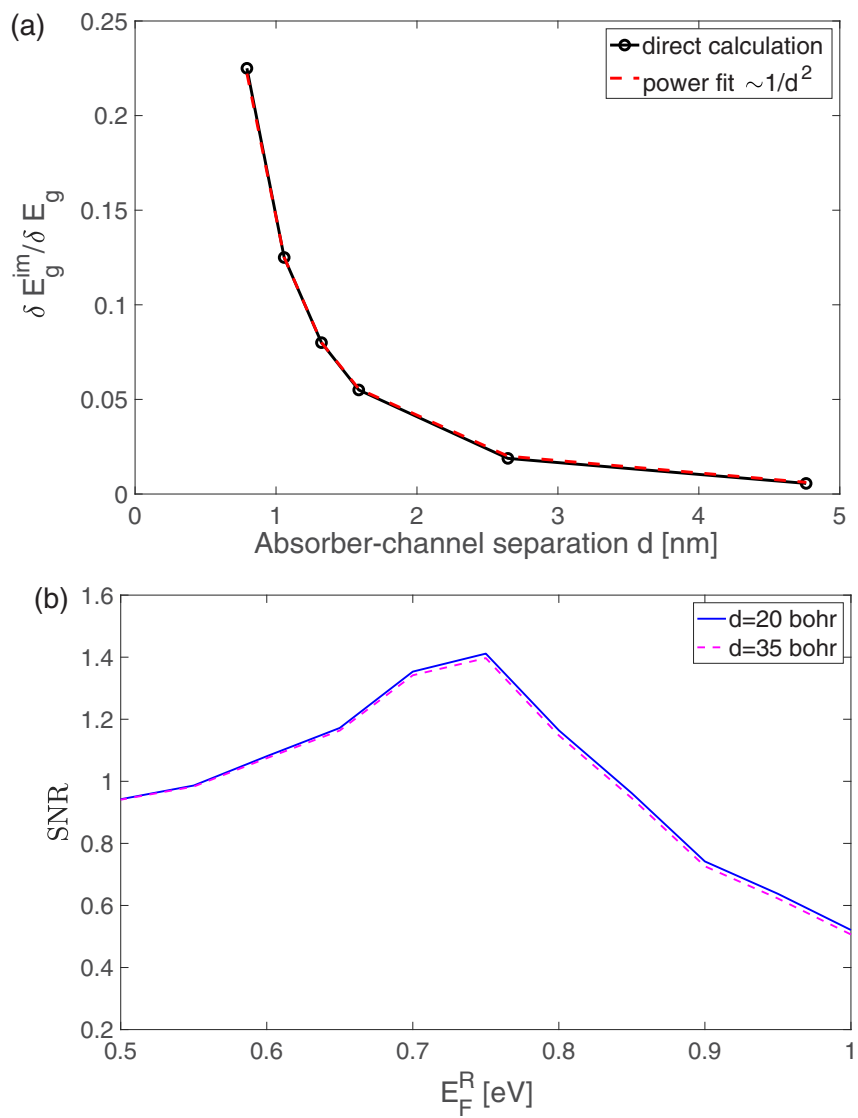

FIG. 8. (a) Ratio $\delta E_{g}^{i m}(t) / \delta E_{g}(t) . E_{F}^{L}$ is well above the channel energy levels and $E_{F}^{R}=0.7 \mathrm{eV}$. (b) SNR results for two different absorber-channel separations: $d=20$ bohrs [same as the solid blue line in Fig. 7(a)] and $d=35$ bohrs. $N=3, j=2 . d$ is defined as the distance between the absorber site farthest away from the channel and the central channel site. Calculations are performed by treating the electron correlation inside the conducting channel at the $G W$ level.

By comparing Eqs. (B5) and (B7), it becomes clear that the two terms display different scaling behavior with respect to the absorber-channel separation $d$. Indeed, $w_{c h}$ on the righthand side of Eq. (B5) is obtained in the absence of the absorber, hence, it does not depend on $d$. By contrast, $\dot{w}$ on the right-hand side of Eq. (B7) becomes negligible at large $d$.

For a quantitative comparison, we calculated the two contributions $\delta E_{g}^{i m}(t)$ and $\delta E_{g}(t)$ using Eqs. (B6) and (B2) for a three-site channel with source-drain and gate voltages that correspond to the optimal SNR for this geometry (see Fig. 7). For consistency, both terms were calculated with the electron correlation inside the conducting channel treated at the $G W$ level. The calculated ratio $\delta E_{g}^{i m}(t) / \delta E_{g}(t)$ is independent of the particular (small) value of $\mathcal{P}(t)$ [as it should be from Eqs. (B5) and (B7)] and the result is shown in Fig. 8(a). As expected, $\delta E_{g}^{i m}(t) / \delta E_{g}(t) \rightarrow 0$ at large $d$ (more exactly we find a $\sim 1 / d^{2}$ behavior). We see that for the typical separation $d \sim 1 \mathrm{~nm}$ the term $\delta E_{g}^{i m}(t)$ is about one order of magnitude smaller than the term $\delta E_{g}(t)$, i.e., it would lead to a $\sim 10 \%$ increase in the channel back-action. This $10 \%$ increase in the back-action leads to less than 5\% decrease in the SNR [57]. 
At larger separations $d>1 \mathrm{~nm}$, the impact of the term $\delta E_{g}^{i m}(t)$ becomes even smaller.

The above can be corroborated with the fact that the SNR [calculated without accounting for $\delta E_{g}^{i m}(t)$ ] is virtually independent of $d$, as seen in Fig. 8(b). This is due to the fact that with increasing absorber-channel separation the reduced Coulomb coupling leads to a decrease in channel back-action that is almost exactly canceled by a corresponding decrease in the absorber action. We note that with increasing distance, one adjusts accordingly the pulse duration: the optimal $\tau_{0}$ increases with $d$, reflecting the smaller channel back-action. The robustness of the SNR against increasing distance holds until the optimal $\tau_{0}$ saturates, when reaching values $\sim \tau_{\text {rad }}$.

Together, the results in Fig. 8 lead us to conclude that the term $\delta E_{g}(t)$ (accounted for in the results presented in the main text) is the dominant renormalization term for $d \gtrsim 1 \mathrm{~nm}$ and that the main conclusion of our work (that it is possible to achieve large SNRs under optimal conditions) is not affected by the neglect of contribution (II).
[1] M. Giustina, Marijn A. M. Versteegh, S. Wengerowsky, J. Handsteiner, A. Hochrainer, K. Phelan, F. Steinlechner, J. Kofler, J.-A. Larsson, C. Abellan, W. Amaya, V. Pruneri, M. W. Mitchell, J. Beyer, T. Gerrits, A. E. Lita, L. K. Shalm, S. W. Nam, T. Scheidl et al., Phys. Rev. Lett. 115, 250401 (2015).

[2] H. Takesue, S. D. Dyer, M. J. Stevens, V. Verma, R. P. Mirin, and S. W. Nam, Optica 2, 832 (2015).

[3] R. H. Hadfield, Nat. Photonics 3, 696 (2009).

[4] S. M. Young, M. Sarovar, and F. Léonard, Phys. Rev. A 98, 063835 (2018).

[5] M. A. Rowea, E. J. Gansen, M. Greene, R. H. Hadfield, T. E. Harvey, M. Y. Su, S. W. Nam, and R. P. Mirin, Appl. Phys. Lett. 89, 253505 (2006).

[6] X. Zhou, T. Zifer, Thomas, B. M. Wong, K. L. Krafcik, F. Léonard, and A. L. Vance, Nano Lett. 9, 1028 (2009).

[7] X. Guo, L. Huang, S. O'Brien, P. Kim, and C. Nuckolls, J. Am. Chem. Soc. 127, 15045 (2005).

[8] D. S. Hecht, R. J. A. Ramirez, M. Briman, E. Artukovic, K. S. Chichak, J. F. Studdard, and G. Gruner, Nano Lett. 6, 2031 (2006).

[9] C. Jia, A. Migliore, N. Xin, S. Huang, J. Wang, Q. Yang, S. Wang, H. Chen, D. Wang, B. Feng, Z. Liu, G. Zhang, D.-H. Qu, H. Tian, M. A. Ratner, H. Q. Xu, A. Nitzan, and X. Guo, Science 352, 1443 (2016).

[10] Y. Gao and M. Galperin, J. Chem. Phys. 144, 174113 (2016).

[11] M. Galperin and A. Nitzan, J. Chem. Phys. 124, 234709 (2006); B. D. Fainberg, M. Jouravlev, and A. Nitzan, Phys. Rev. B 76, 245329 (2007).

[12] D. A. Stewart and F. Léonard, Phys. Rev. Lett. 93, 107401 (2004).

[13] F. Léonard, C. D. Spataru, M. Goldflam, D. W. Peters, and T. E. Beechem, Sci. Rep. 7, 45873 (2017).

[14] S. M. Young, M. Sarovar, and F. Léonard, Phys. Rev. A 97, 033836 (2018).

[15] M. Stobińska, G. Alber, and G. Leuchs, Europhys. Lett. 86, 14007 (2009).

[16] Y. Wang, J. Minář, L. Sheridan, and V. Scarani, Phys. Rev. A 83, 063842 (2011).

[17] J. E. Gough, M. R. James, H. I. Nurdin, and J. Combes, Phys. Rev. A 86, 043819 (2012).

[18] Other configurations such as waveguides can also be treated with simple modifications. See P. Domokos, P. Horak, and H. Ritsch, Phys. Rev A 65, 033832 (2002).

[19] C. D. Spataru, S. Ismail-Beigi, L. X. Benedict, and S. G. Louie, Phys. Rev. Lett. 92, 077402 (2004).

[20] R. B. Capaz, C. D. Spataru, S. Ismail-Beigi, and S. G. Louie, Phys. Status Solidi B 244, 4016 (2007).
[21] S. J. Strickler and R. A. Berg, J. Chem. Phys. 37, 814 (1962).

[22] R. Loudon, The Quantum Theory of Light, 3rd ed. (Oxford University Press, Oxford, 2000).

[23] D. A. Steck, Quantum and Atom Optics, available online at http://steck.us/teaching (revision 0.12.6, 23 April 2019).

[24] K. Ohno, Theor. Chim. Acta 2, 219 (1964).

[25] Additional care needs to be paid when designing a realistic device where the conducting channel is placed on top of a substrate: the electronic level alignment and/or separation between absorber and substrate need to be judiciously chosen to minimize energy transfer losses.

[26] C. D. Spataru and F. Léonard, Phys. Rev. Lett. 104, 177402 (2010); Chem. Phys. 413, 81 (2013).

[27] S. Latini, E. Perfetto, A.-M. Uimonen, R. van Leeuwen, and G. Stefanucci, Phys. Rev. B 89, 075306 (2014).

[28] M. S. Hybertsen and S. G. Louie, Phys. Rev. B 34, 5390 (1986).

[29] A.-P. Jauho, N. S. Wingreen, and Y. Meir, Phys. Rev. B 50, 5528 (1994).

[30] For the particular case of a coherent light pulse the OBE approach is equivalent to more general approaches such as the quantized propagating pulse [16] or master-equation [17] approaches.

[31] For $N=7$ and the design that yields $\mathrm{SNR} \approx 4.5$ we estimate $F=0.35$ from $F=\int d E\left(f_{L}-f_{R}\right) T(1-T) / \int d E\left(f_{L}-f_{R}\right) T$ where $T(E)$ is the calculated Landauer transmission. This estimate, however, does not account for the impact of the absorber on current fluctuations.

[32] Y. M. Blanter and M. Buttiker, Phys. Rep. 336, 1 (1999).

[33] L. S. Levitov and G. B. Lesovik, Pis'ma Zh. Eksp. Teor. Fiz. 55, 534 (1992) [JETP Lett. 55, 555 (1992)]; Pis'ma Zh. Eksp. Teor. Fiz. 58, 225 (1993) [JETP Lett. 58, 230 (1993)].

[34] Yu. V. Nazarov and M. Kindermann, Eur. Phys. J. B 35, 413 (2003).

[35] L. V. Keldysh, Zh. Eksp. Teor. Fiz. 47, 1515 (1965) [Sov. Phys.JETP 20, 1018 (1965)].

[36] C. Attaccalite, M. Gruning, and A. Marini, Phys. Rev. B 84, 245110 (2011).

[37] D. C. Langreth, in Linear and Nonlinear Electron Transport in Solids, edited by J. T. Devresse and E. Van Doren (Plenum, New York, 1976).

[38] H. Haug and A.-P. Jauho, Quantum Kinetics in Transport and Optics of Semiconductors (Springer, Berlin, 1998).

[39] K. Balzer and M. Bonitz, Nonequilibrium Green's Functions Approach to Inhomogeneous Systems, Lecture Notes in Physics (Springer, Berlin, 2013). 
[40] V. Spicka, B. Velicky, and A. Kalvova, Int. J. Mod. Phys. B 28, 1430013 (2014).

[41] L. P. Kadanoff and G. Baym, Quantum Statistical Mechanics: Green's Function Methods in Equilibrium and Nonequilibrium Problems (Addison-Wesley, Boston, 1989).

[42] P. Lipavsky, V. Spicka, and B. Velicky, Phys. Rev. B 34, 6933 (1986).

[43] In practice, the NEGF dynamics is obtained by treating the interaction between the absorber and the transport channel at the Hartree level (the exchange Fock self-energy vanishes in the absence of charge transfer between absorber and transport channel). Because the absorber accommodates a single electron, there are no Coulomb interaction effects that need to be accounted for inside the absorber subspace. Inside the transport channel subspace we include spin degeneracy but neglect Coulomb interaction effects. The impact of electron correlation effects beyond the Hartree-Fock level is studied within the $G W$ approximation in Appendix B.

[44] C. Runge, Math. Ann. 46, 167 (1895).

[45] P. Myohanen, R. Tuovinen, T. Korhonen, G. Stefanucci, and R. van Leeuwen, Phys. Rev. B 85, 075105 (2012).

[46] C. D. Spataru, M. S. Hybertsen, S. G. Louie, and A. J. Millis, Phys. Rev. B 79, 155110 (2009).

[47] X. Wang, C. D. Spataru, M. S. Hybertsen, and A. J. Millis, Phys. Rev. B 77, 045119 (2008).

[48] C. D. Spataru, Phys. Rev. B 82, 195111 (2010).

[49] C. Hwang, D. A. Siegel, S.-K. Mo, W. Regan, A. Ismach, Y. Zhang, A. Zettl, and A. Lanzara, Sci. Rep. 2, 590 (2012).

[50] A. Bostwick, F. Speck, T. Seyller, K. Horn, M. Polini, R. Asgari, A. H. MacDonald, and E. Rotenberg, Science 328, 999 (2010).
[51] By that we mean that the excited dipole is small enough such that the renormalization of the absorber optical gap remains well within the bounds set by the photon bandwidth (see inset of Fig. 2 in the main text).

[52] J. M. Garcia-Lastra and K. S. Thygesen, Phys. Rev. Lett. 106, 187402 (2011).

[53] C. D. Spataru, Phys. Rev. B 88, 125412 (2013)).

[54] The absorber optical level also gets renormalized via the interaction between the absorber transition dipole and its image in the channel (see Ref. [53]). In this case the renormalization is $\sim\left\langle\phi_{g}^{*} \phi_{e}\left|w_{c h}\left(E_{g}\right)-v\right| \phi_{e}^{*} \phi_{g}\right\rangle$ where the relevant frequency for the channel screening is that of the optical gap. Because the optical gap of our absorber $(\approx 7.5 \mathrm{eV})$ is much larger than the channel bandwidth $(\approx 2 \mathrm{eV})$, this effect (captured at the Hartree-Fock level) is negligible.

[55] J. B. Neaton, Mark S. Hybertsen, and Steven G. Louie, Phys. Rev. Lett. 97, 216405 (2006).

[56] By $t=0$ we mean the initial times (when the photon field starts interacting with the absorber) where the renormalization of the absorber optical gap can be neglected. The corresponding timescale is of course larger than the $\sim$ fs light period.

[57] The inclusion of electron correlation effects between channel and absorber affects not only the channel back-action, but also the absorber action (the absorber renormalizes the quasiparticle energies in the channel, hence the current, via a similar image charge effect as discussed previously, where the roles of the absorber and channel are interchanged). While for the sake of brevity we do not address the change in absorber action here, we expect [e.g., based on the results shown in Fig. 8(b)] that it tends to cancel the change in channel back-action with an overall impact of electron correlation effects on the SNR even smaller than $5 \%$. 